
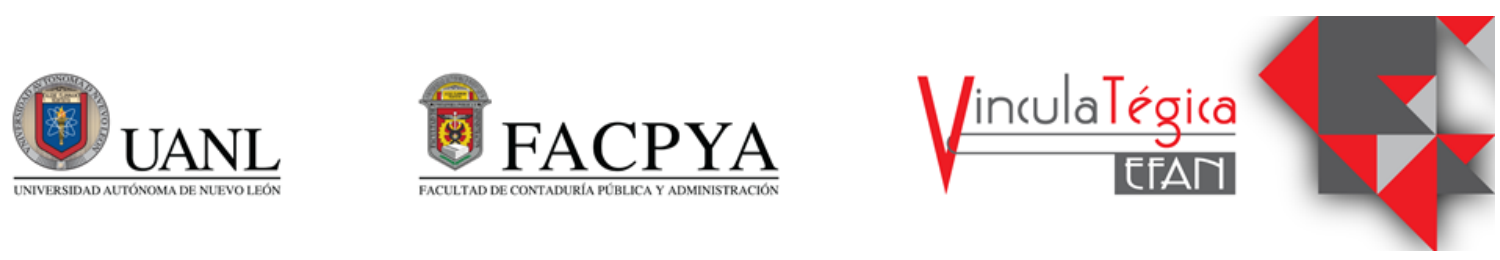

\title{
El etnocentrismo como antecedente de la intención de compra de productos nacionales
}

\author{
Alexis Jazmín Morán Huertas ${ }^{1}$, Alfoso López-Lira² y Mayela Terán Cazares ${ }^{3}$ \\ ${ }^{1}$ Universidad Autónoma de Nuevo León, Facultad de Contaduría Pública y Administración, \\ alexis.moranhr@uanl.edu.mx,Av.Universidad S/N Col.Ciudad Universitaria, 8187998150 \\ ${ }^{2}$ Universidad Autónoma de Nuevo León, Facultad de Contaduría Pública y Administración, \\ alfonso.lopezlr@uanl.edu.mx,Av.Universidad S/N Col.Ciudad Universitaria. \\ ${ }^{3}$ Universidad Autónoma de Nuevo León, Facultad de Contaduría Pública y Administración, \\ mayela.teran@gmail.com, Av.Universidad S/N Col. Ciudad Universitaria.
}

\author{
Información del artículo revisado por pares \\ Fecha de aceptación: junio-2021 \\ Fecha de publicación en línea: diciembre-2021 \\ DOI: https://doi.org/10.29105/vtga7.2-57
}

\begin{abstract}
Resumen
El objetivo de esta investigación es identificar los principales estudios que demuestran que la variable etnocentrismo es un antecedente de la intención de compra de productos nacionales, con la finalidad de que en futuros estudios se compruebe si en México la relación entre variables es significativa. En esta investigación cualitativa se seleccionaron 22 artículos en total para el desarrollo de este artículo. Se encontró teóricamente en los estudios empíricos que el etnocentrismo es un antecedente de la intención de compra de productos nacionales y muestra una relación significativa con la variable dependiente. Se recomienda que las futuras líneas de investigación realicen estudios empíricos en México y distintas regiones de Latinoamérica para medir estadísticamente el grado de influencia de los consumidores en la intención de compra de productos nacionales.
\end{abstract}

Palabras clave: Etnocentrismo, intención de compra, productos nacionales.

\begin{abstract}
The objective of this research is to identify the main studies that show that the ethnocentrism variable is an antecedent of the intention to purchase national products, with the aim that future studies will verify whether the relationship between variables is significant in Mexico. In this qualitative research, a total of 22 articles were selected for the development of this article. It was theoretically found in empirical studies that ethnocentrism is an antecedent of the intention to purchase national products and shows a significant relationship with the dependent variable. It is recommended that future lines of research carry out empirical studies in Mexico and different regions of Latin America to statistically measure the degree of
\end{abstract}


influence of consumers on the intention to purchase national products.

\section{JEL: M160, M310, M370.}

\section{INTRODUCCIÓN}

La cultura es uno de los elementos externos que más influyen en el comportamiento del consumidor (Jiménez y Gutiérrez, 2010). La cultura se refiere a normas o patrones de comportamiento ampliamente compartidos de un gran grupo de personas, y se define como los valores, actitudes, creencias, artefactos y otros símbolos significativos que representan el patrón de vida adoptado por las personas que los ayudan a interpretar, evaluar y comunicarse, como miembros de la sociedad (Kumar, 2000).

Por consiguiente, es importante que se estudien variables relacionadas con las percepciones y creencias que tienen los consumidores con respecto a su propio país. Estas variables están relacionadas con la cultura que los consumidores han desarrollado a través del tiempo.

Hoy en día, los consumidores tienen acceso a productos de distintos países, por lo tanto, los niveles de etnocentrismo de los consumidores han ido disminuyendo con el paso de los años y han adquirido una mentalidad más abierta y propensión a comprar productos de distintos orígenes.
Keywords: Ethnocentrism, purchase intention, local products.

JEL: M160, M310, M370.
Por lo tanto, esta investigación cualitativa tiene como objetivo general identificar los estudios empíricos en donde el etnocentrismo presenta una influencia positiva y significativa en la intención de compra de productos nacionales. Esto con la finalidad de comprobar, en estudios futuros, si la relación entre estas variables es significativa en los consumidores de México.

Razones por las cuales, la pregunta de investigación que se busca responder de manera teórica es: ¿El etnocentrismo influye positivamente en la intención de compra de productos nacionales? Por lo que, responder esta pregunta de investigación contribuye en la aportación de conocimiento que se comparte con la sociedad.

\section{MARCO TEÓRICO}

\subsection{Etnocentrismo del consumidor}

De acuerdo con Ruyter, Van Birgelen y Wetzels (1998) el etnocentrismo es un concepto, que tiene sus raíces en la sociología, conduce a una evaluación nacionalista de productos extranjeros y ante la creciente internacionalización, el etnocentrismo del consumidor puede jugar un papel importante en el 
mercado global, ya que los consumidores etnocéntricosson reacios a hacer uso de los productos extranjeros, debido a un sentido de lealtad hacia su país de origen y, a su vez, estas tendencias etnocéntricas del consumidor pueden llevar a las actitudes negativas de los productos extranjeros.

Shimp y Sharma (1987), utilizaron el término etnocentrismo del consumidor para representar las creencias de los consumidores acerca de lo apropiado e incluso lo moral de comprar productos hechos en el extranjero.

De acuerdo con Sharma, Shimp y Shin (1995), las características del etnocentrismo del consumidor residen en la preocupación por el propio país y el temor de someter los propios intereses económicos por los resultados y efectos nocivos que las importaciones pueden traer.

Jiménez y Gutiérrez (2010), mencionan que el etnocentrismo se refiere a la preferencia del consumidor por los productos nacionales sobre los extranjeros. Kerin et al. (2014) explica los consumidores etnocéntricos creen que la compra de productos importados es mala por ser antipatriótica, por dañar las industrias nacionales y producir desempleo en su país.

Czinkota y Ronkainen (2008), define el etnocentrismo como la creencia de que la cultura propia es superior a las demás. Shiffman y Lazar (2010), menciona que el etnocentrismo es un reflejo de la personalidad del consumidor, ya que refleja el temor o rechazo a comprar un producto de manufactura extranjera. De acuerdo con Keegan y Green (2009), una persona que asume que su país de origen es superior al resto del mundo tiene una orientación etnocéntrica

De acuerdo con algunos autores, los consumidores altamente etnocéntricos tendrán actitudes negativas hacia los productos importados y actitudes positivas hacia los productos locales (Sharma et al., 1995; Watson y Wright, 2000).

El etnocentrismo del consumidor significa la idoneidad, de hecho, la moralidad de la compra productos fabricados en el extranjero (Bawa, 2004).

El etnocentrismo del consumidor implica que comprar importaciones está mal porque es antipatriótico y perjudicial para el hogar, economía y empleo (Supphellen y Rittenburg (2001). Las personas etnocéntricas creen que su propio grupo es el centro de su mundo y utilizarán su propia cultura como comparador.

El etnocentrismo se extiende al campo del marketing cuando se consideran los factores que influyen $y$ forjar el comportamiento del consumidor (Javalgi, Khare, Gross, y Scherer, 2005).

La literatura describe el concepto de etnocentrismo del consumidor como un medio para comprender las preocupaciones morales derivados del consumo de productos nacionales y extranjeros productos (Siamagka y Balabanis, 2015). 
El etnocentrismo del consumidor es una actitud del consumidor preocupada por sentimientos proteccionistas a nivel individual, y ha sido ampliamente investigado por investigadores de marketing. (Bizumic, 2019).

Luque-Martínez, Ibáñez-Zapata y BarrioGarcía (2000) mencionan que la investigación sobre el etnocentrismo del consumidor puede ser un paso importante hacia una mejor comprensión de la forma en que los consumidores comparan los productos nacionales con los extranjeros, así como las razones por las que llevarlos a desarrollar prejuicios patrióticos contra las importaciones.

Los consumidores altamente etnocéntricos tienden a emitir juicios sesgados, por lo que sobre evalúan productos nacionales irrazonablemente, en comparación con los productos importados, es decir, los consumidores tienden a enfatizar los aspectos positivos de los productos nacionales y a infravalorar las virtudes de los productos extranjeros (Luque-Martínez, Ibáñez-Zapata y Barrio-García, 2000).

\subsection{Intención de compra de productos nacionales}

En este estudio la intención de compra de productos nacionales se define como el grado de disposición, por parte de los consumidores, para comprar un producto.

De acuerdo con los autores Ajzen y Fishbein (1977) la intención de compra del consumidor sirve para predecir la compra real.

\subsection{Estudios empíricos del etnocentrismo y la intención de compra}

Shimp y Sharma (1987), desarrollaron una escala de 17 reactivos con una fiabilidad muy alta, lo que respalda la consistencia interna de la escala. Los resultados indicaron que el etnocentrismo tiene una correlación negativa con la intención de compra de productos extranjeros, argumentando que el etnocentrismo del consumidor afecta la intención de compra de productos locales y de productos importados, y que es un predictor relevante de actitudes, intenciones $y$ comportamiento de compra de los productos extranjeros.

Además, encontraron que las personas mayores de la clase obrera manifestaron tendencias etnocéntricas particularmente fuertes debido a que están especialmente amenazadas por las perspectivas de perder empleos ante competidores extranjeros y no poder encontrar otros nuevos, mientras que los trabajadores de clase media y media alta, especialmente los últimos, tienen empleos relativamente más seguros y sus tendencias etnocéntricas son particularmente débiles (Shimp y Sharma, 1987).

Rybina et al. (2010), encontraron que el etnocentrismo del consumidor de Kazakhstan conduce a un alto consumo de bienes producidos localmente y a un menor nivel de consumo de productos fabricados en el extranjero.

Awdziej, Wlodarek y Tkaczyk (2016), obtuvieron que el etnocentrismo tiene una 
relación negativa con la intención de compra de productos extranjeros. Para su estudio utilizaron comida y midieron la relación entre el nivel de etnocentrismo y la edad.

Por lo que en adultos jóvenes (20-25 años) y adultos (26-59 años) el nivel de etnocentrismo fue bajo, mientras que en los adultos mayores (más de 60 años) el nivel de etnocentrismo fue alto, y a pesar de esto, la correlación entre edad y etnocentrismo fue muy baja (Awdziej, Wlodarek y Tkaczyk, 2016).

Los resultados de Shoham y Makovec (2003), indicaron que el etnocentrismo del consumidor de Israel fue un predictor significativo de la intención de compra de productos extranjeros, en este caso productos de Israel, por lo que al tener niveles altos de etnocentrismo preferían comprar productos locales.

Zeugner-Roth et al. (2015), encontraron que el etnocentrismo tiene un impacto significativo negativo en la intención de compra de productos extranjeros.

Las muestras se obtuvieron de Austria y Eslovenia, utilizando productos italianos, y con su análisis se reveló que la muestra de Austria fue más etnocéntrica que la muestra de Eslovenia, que fue más cosmopolita (Zeugner-Roth et al., 2015).

Yoo y Donthu (2005), obtuvieron que el etnocentrismo del consumidor estadounidense esta negativamente correlacionado con la intención de compra de productos extranjeros (en su caso utilizaron productos japoneses tales como televisiones, radio, cámaras, refrigeradores) y la correlación fue significativa.

En cambio, Jiménez y Gutiérrez (2010), demostraron que el etnocentrismo no influye significativamente en la intención de compra, por lo que su hipótesis el etnocentrismo del consumidor influye negativamente en su intención de compra de productos extranjeros, se rechazó.

Además, encontraron que en los consumidores más jóvenes el etnocentrismo disminuye y no reduce la intención de compra, mientras que en las personas mayores el etnocentrismo aumenta y reduce la intención de compra de productos extranjeros (Jiménez y Gutiérrez, 2010).

\section{MÉTODO}

Esta investigación fue cualitativa debido a que se realizó un análisis teórico de la literatura para encontrar hallazgos empíricos que demostraran la relación entre el etnocentrismo y la intención de compra de productos nacionales.

La literatura que se revisó fue de libros, artículos científicos, tesis doctorales, entre otras, relacionados con el área de mercadotecnia y negocios internacionales. $\mathrm{Se}$ revisaron aproximadamente 125 artículos de los cuales se seleccionaron aquellos que estuvieran relacionados con el tema de investigación, que la variable etnocentrismo tuviera un impacto 
significativo en la variable dependiente intención de compra de productos nacionales y que fueran publicados en revistas científicas de alto impacto. Finalmente, se seleccionaron 22 artículos en total.

Uno los criterios para la selección de los artículos fue que las revistas fueran de alto impacto, actuales y que incluyeran variables independientes relacionadas con el área de la psicología y la sociología y que, además, tuvieran una influencia positiva en la intención de compra de productos extranjeros, esto con la finalidad de cumplir con el objetivo de investigación.

Considerando que la investigación fue cualitativa, la clasificación de la unidad de análisis para la búsqueda de los estudios empíricos fue a nivel individual. Al ser una investigación cualitativa, el análisis de la literatura fue teórico, por lo tanto, se describió y analizó la literatura de diferentes autores mediante un proceso inductivo, definiendo variables y demostrando su relación mediante artículos empíricos.

\section{RESULTADOS}

Una vez revisada la literatura con relación a las definiciones y estudios empíricos que demostraron una relación significativa de la variable etnocentrismo en la variable dependiente intención de compra de productos extranjeros se encontró si existe una relación significativa entre estas dos variables.

Tabla 1. Estudios empíricos de la influencia del etnocentrismo en la intención de compra de productos nacionales.

Autores $\quad$ Variable independiente $\quad$ Relación con la variable dependiente

Shimp y Sharma (1987) Etnocentrismo $\quad$ Significativa

$\begin{array}{ll}\text { Rybina et al. (2010) } \quad \text { Etnocentrismo } & \text { Significativa }\end{array}$

$\begin{array}{lll}\text { Awdziej, Wlodarek y Tkaczyk } & \text { Etnocentrismo } & \text { Significativa }\end{array}$ (2016)

$\begin{array}{lll}\text { Shoham y Makovec (2003) } & \text { Etnocentrismo } & \text { Significativa }\end{array}$

$\begin{array}{lll}\text { Zeugner-Roth et al. (2015) Etnocentrismo } & \text { Significativa }\end{array}$

$\begin{array}{lll}\text { Yoo y Donthu (2005) } & \text { Etnocentrismo } & \text { Significativa }\end{array}$ 
Fuente: Elaboración propia.

Las hipótesis de investigación generadas como resultado del análisis teórico fueron las siguiente:

- $\mathrm{H}_{1}$ : El etnocentrismo influye positivamente en la intención de compra de productos nacionales.

- $\mathrm{H}_{2}$ : El xenocentrismo influye negativamente en la intención de compra de productos nacionales.

En la Tabla 1, se resumen los estudios empíricos que demuestran una relación significativa con la variable dependiente intención de compra de productos nacionales como resultado del análisis teórico. Por lo tanto, estudiar dicha relación es importante para comprobar si en una muestra mexicana la relación es significativa.

\section{CONCLUSIONES}

En esta investigación se cumplió con el objetivo de estudio, ya que se encontraron estudios empíricos que demuestran la relación entre la variable etnocentrismo y la intención de compra de productos extranjeros.

Se recomienda que en futuras investigaciones se realicen estudios empíricos que demuestren estadísticamente la relación entre variables para responder con argumentos estadísticos a la pregunta de investigación.

Así mismo, los estudios en economías emergentes son de gran relevancia teórica, por lo que aplicar un instrumento de medición en México o en algunas regiones de América Latina sería de gran relevancia para la aportación de conocimiento. 


\section{REFERENCIAS}

Ajzen, I., \& Fishbein, M. (1977). Attitude-Behavior Relations: A Theoretical Analysis and Review of Empirical Research. Psychological Bulletin, 84(5), 888-918. doi: 10.1007/s11614-012-0060-4

Ajzen, I., \& Fishbein, M. (1977). Attitude-Behavior Relations: A Theoretical Analysis and Review of Empirical Research. Psychological Bulletin, 84(5), 888-918. doi: 10.1007/s11614-012-0060-4

Awdziej, M., Wlodarek, D., \& Tkaczyk, J. (2016). Are elderly consumer more ethnocentric? Attitudes towards Polish and'foreign'food products. Journal of Economics \& Management, 23, 91.

Bizumic, B. (2019). Effects of the dimensions of ethnocentrism on consumer ethnocentrism. International Marketing Review.

Czinkota, M. R., \& Ronkainen, I. A. (2008). Marketing internacional (8 ed.). México: Cengage Learning.

De Ruyter, K., Van Birgelen, M., \& Wetzels, M. (1998). Consumer ethnocentrism in international services marketing. International Business Review, 7(2), 185-202.

Javalgi, R. G., Khare, V. P., Gross, A. C., \& Scherer, R. F. (2005). An application of the consumer ethnocentrism model to French consumers. International Business Review, 14(3), 325-344.

Jiménez, N., \& Gutiérrez, S. (2010). Antecedentes socio-psicológicos de la intención de compra de productos extranjeros: el etnocentrismo y la animosidad del consumidor. Revista Española de Investigación de Marketing, 14(1), 7-24.

Keegan, W. \& Green, M. (2009). Marketing internacional. Prentice Hall.

Kerin, R., Rudelius, W., \& Hartley, S. (2014). Marketing. México: McGraw Hill.

Kumar, V. (2000), International Marketing Research. Upper Saddle River, NJ: Prentice Hall.

Luque-Martínez, T., Ibáñez-Zapata, J. A., \& del Barrio-García, S. (2000). Consumer ethnocentrism measurement-An assessment of the reliability and validity of the CETSCALE in Spain. European Journal of Marketing.

Rybina, L., Reardon, J., \& Humphrey, J. (2010). PATRIOTISM, COSMOPOLITANISM, CONSUMER ETHNOCENTRISM AND PURCHASE BEHAVIOR IN KAZAKHSTAN. Organizations \& Markets In Emerging Economies, 1(2), 92-107. 
Shankarmahesh, M. N. (2006). Consumer ethnocentrism: an integrative review of its antecedents and consequences. International marketing review.

Sharma, S., Shimp, T., \& Shin, J. (1995). Consumer ethnocentrism: A test of antecedents and moderators. Journal of the academy of marketing science, 23(1), 26-37.

Shimp, T., \& Sharma, S. (1987). Consumer Ethnocentrism: Construction and Validation of the CETSCALE. Journal Of Marketing Research (JMR), 24(3), 280-289.

Shimp, T., \& Sharma, S. (1987). Consumer Ethnocentrism: Construction and Validation of the CETSCALE. Journal Of Marketing Research (JMR), 24(3), 280-289.

Shoham, A., \& Makovec, M. (2003). Consumer ethnocentrism, attitudes, and purchase behavior: An Israeli study. Journal of International Consumer Marketing, 67-86.

Siamagka, N. T., \& Balabanis, G. (2015). Revisiting consumer ethnocentrism: review, reconceptualization, and empirical testing. Journal of International Marketing, 23(3), 66-86.

Supphellen, M., \& Rittenburg, T. L. (2001). Consumer ethnocentrism when foreign products are better. Psychology \& Marketing, 18(9), 907-927.

Watson, J. \& Wright, K. (2000). "Consumer Ethnocentrism and Attitudes Toward Domestic and Foreign Products," European Journal of Marketing, 34 (9/10), 1149-1166.

Yoo, B., \& Donthu, N. (2005). The effect of personal cultural orientation on consumer ethnocentrism: Evaluations and behaviors of US consumers toward Japanese products. Journal of International Consumer Marketing, 18(1-2), 7-44.

Zeugner-Roth, K., Žabkar, V. \& Diamantopoulos, A. (2015). Consumer Ethnocentrism, National Identity, and Consumer Cosmopolitanism as Drivers of Consumer Behavior: A Social Identity Theory Perspective. Journal Of International Marketing, 25-54, 36. 